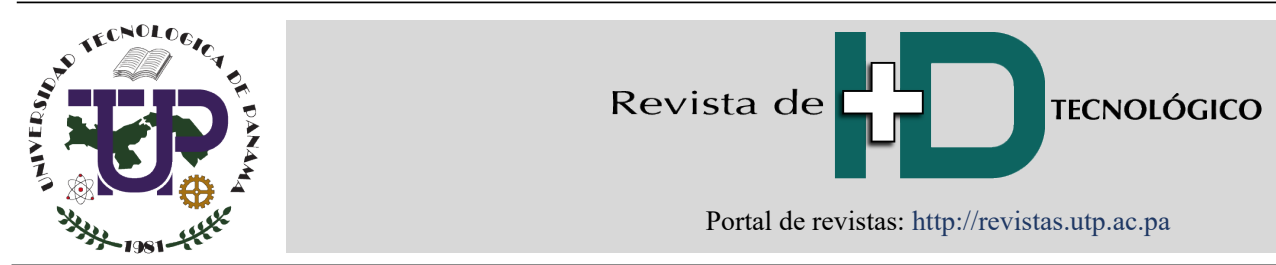

\title{
Resiliencia de Sistemas Eléctricos de Potencia mediante la Conmutación de Líneas de Transmisión - Estado del arte
}

\section{Electrical Power Systems Resilience by Switching of Power Transmission Lines - State of art}

\author{
Jaime Pilatásig Lasluisa ${ }^{1 *}$, Diego Carrión ${ }^{1}$ \\ ${ }^{1}$ Ingeniería Eléctrica, Universidad Politécnica Salesiana, Ecuador \\ *Autor de correspondencia: jpilatasigl@est.ups.edu.ec
}

RESUMEN- Los sistemas eléctricos se ven amenazados por diversos factores que afectan la continuidad en el suministro de electricidad, a la economía y a las personas; los eventos extremos tanto derivados del cambio climático o de los desastres naturales, identificados como riesgos de baja probabilidad, pero de alto impacto han causado grandes apagones a nivel mundial. La resiliencia es un aspecto que define la capacidad de los sistemas de energía para recuperarse de los disturbios sean de corta o larga duración. Se aborda la conmutación de líneas de transmisión para incrementar la resiliencia del sistema y reducir costos totales en el contexto del despacho económico. Se resalta los aspectos considerados en las publicaciones que abarcan la temática de la resiliencia en los sistemas eléctricos, la conmutación de las líneas de transmisión bajo escenarios de evaluación de contingencia N-1, y, la planificación de expansión de transmisión comprendiendo los planteamientos de distintos autores y sus conclusiones. En la literatura técnica revisada, se visualiza el alcance de sus resultados para direccionarlos para el estudio de la Restauración Óptima de la Operación de Sistemas Eléctricos de Potencia mediante la Conmutación de Líneas de Transmisión. Se determina que el punto de partida es el flujo óptimo de potencia DC (DCOPF), encontrándose la mejor solución y sobre todo la minimización de los costos totales de despacho.

Palabras clave-Resiliencia, líneas de transmisión, conmutación de líneas de transmisión, sistemas eléctricos de potencia, operación óptima, flujos óptimos de potencia, planificación de expansión de transmisión.

ABSTRACT- Power systems are threatened by various factors that affect the continuity in the supply of electricity, the economy and people; extreme events, derived from climate change or from natural disasters, identified as low probability but high impact risks have caused major blackouts worldwide. Resilience is an aspect that defines the ability of energy systems to recover from short or long-term disturbances. The switching of transmission lines is addressed to increase the resilience of the system and reduce total costs in the context of the economic dispatch.

It highlights the aspects considered in the publications that cover the theme of resilience in the power systems, the transmission lines switching under scenarios of contingency evaluation $\mathrm{N}-1$, and, transmission expansion planning including the approaches of different authors and their conclusions. In the revised technical literature, the scopes of their results are highlighted to direct them for the study of the Optimal Restoration of the Power Systems Operation by means of Transmission Lines Switching. From which it is determined that the starting point is the Dc optimal power flow (DCOPF), finding the best solution and the minimization of the total costs of dispatch.

Keywords-Resilience, climate change, transmission lines, switching, modeling, optimization, cost, transmission expansion planning.

\section{Introducción}

Los sistemas eléctricos están expuestos a sufrir perturbaciones severas que pueden llevar a su colapso, siendo así que el número y duración de las desconexiones son afectadas por factores ambientales, físicos $\mathrm{y}$ tecnológicos [1], los eventos relacionados al clima extremo están considerados dentro de las principales causas que influyen significativamente en la confiabilidad y operación de los componentes del sistema eléctrico [2]-[5], los desastres naturales en más de una

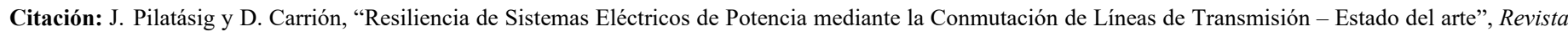
de I+D Tecnológico, vol. 16, no. 2, pp. (no_modificar), 2020.

Tipo de artículo: Original. Recibido: 25 de octubre de 2019. Recibido con correcciones: 25 de octubre de 2019. Aceptado: 7 de julio de 2020.

DOI.

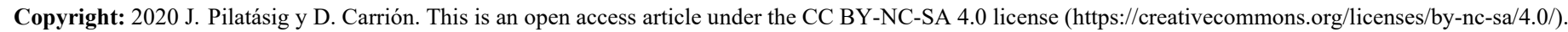


ocasión han comprometido el suministro de electricidad [6]. Este impacto se evidencia en la sociedad y en la economía, por lo que se ha encaminado varias investigaciones sobre la capacidad de restaurar los servicios de energía inmediatamente [7], buscándose determinar varias estrategias o modelos para mejorar la resiliencia de los sistemas eléctricos.

Cada día los sistemas eléctricos se vuelven más complejos, se integran energías renovables no convencionales, nuevas tecnologías, con lo cual se han consolidado los aspectos relacionados a la generación distribuida, redes inteligentes, que deben ser analizadas integralmente con el sistema eléctrico, de este modo buscando mejorar la resiliencia ante cualquier evento.

En [8], considera siete indicadores clave para la resiliencia de un sistema eléctrico, siendo: a) la dependencia del combustible no renovable en la generación, b) la eficiencia de la generación que pretende el balance de la energía requerida versus la energía generada, c) la eficiencia de la distribución, que incluye las pérdidas en transmisión y distribución así como el consumo en la industria, d) la intensidad de dióxido de carbono en la generación, la dependencia de combustibles fósiles en la generación incrementará los costos de producción y el precio del cambio climático, e) diversidad en la generación, es decir, la pertinencia de que la generación de electricidad considere diversos tipos de combustibles o fuentes de energía, f) Potencia redundante para disponer de capacidad para responder ante eventos de alta probabilidad y bajo impacto, g) confianza en la importación, para aquellos países que dependen de la importación de electricidad.

Un sistema de potencia resiliente debe ser capaz de ejecutar acciones preventivas, mitigar el impacto de eventos extremos, responder de forma óptima a través de procedimientos de control automatizados, reducir el tiempo para restablecer el servicio de electricidad y propender a ahorros económicos significantes, según [9].

Un componente importante de un sistema eléctrico, y enlace entre la generación y la carga son las líneas eléctricas, que de igual manera son susceptibles a diversos riesgos, que de materializarse el impacto es sumamente alto; en este sentido, se explora diversos métodos para maximizar la resiliencia del sistema eléctrico a través de la conmutación de líneas de transmisión.
El método tradicional para evaluar la confiabilidad se basa en la simulación de Monte Carlo (MCS), en [10], se presenta un método para clasificación de contingencia que se incorpora para evaluar la confiabilidad de la operación de conmutación de líneas. De lo cual se obtiene un listado de clasificación de líneas recomendadas, líneas seguras y líneas críticas resultantes del análisis de índices anuales y de eventos, determinando que la conmutación de las líneas recomendadas mejora la confiabilidad del sistema.

El punto de partida, son los resultados obtenidos en las investigaciones consideradas, validando sus planteamientos y en gran parte buscando su integración.

Este documento resume los principales esfuerzos de la investigación reciente en la resiliencia de los sistemas eléctricos y se enfoca en los aspectos de la conmutación de líneas de transmisión. Siendo la sección 2 donde se presenta los conceptos y los aspectos de la resiliencia, su interrelación con los eventos externos extremos, derivados del cambio climático y de los desastres naturales, principalmente; la sección 3 enfatiza en la conmutación de líneas de transmisión desde los aspectos de mejorar la resiliencia del sistema de potencia hasta la reducción de costos de despacho de generación, asimismo, se estudia la inclusión del análisis de contingencia N-1 en tiempo real (RTCA); la sección 4 se presentan las conclusiones más relevantes de las contribuciones consideradas en este documento.

\section{Metodología}

\subsection{Resiliencia en sistemas de potencia}

Los modelos globales del clima (GCM) usan un sistema de ecuaciones para representar muchos aspectos del clima, estos aspectos permiten determinar los impactos de eventos de clima extremo en los sistemas eléctricos; los componentes de los sistemas eléctricos se ven amenazados a nivel mundial por el cambio climático, de las recientes investigaciones se determinan impactos como el incremento de la demanda de electricidad, afectación en la capacidad efectiva y eficiencia de generadores térmicos, disminución de los recursos potenciales hidráulicos, de viento y sol para la generación de electricidad y la reducción de la capacidad de transmisión [11].

Para [12], existen varios artículos técnicos que estudian sobre los riesgos que afectarían las infraestructuras críticas de energía en relación con el 
impacto del cambio climático y los fenómenos meteorológicos extremos, en contraste, sólo unos pocos artículos consideran la resiliencia e interdependencia con otros sistemas.

A nivel mundial, de la energía generada entre el $60 \%$ y $80 \%$ se consume en las áreas urbanas, de ahí que la continuidad del suministro de electricidad se ve afectada por el cambio climático y por otras amenazas (ataques cibernéticos, terrorismo, deficiencias técnicas, mercados inestables), afectando a la sociedad en los aspectos económicos y de la vida diaria [13]. Conceptualmente se propone que un sistema resiliente debe ser capaz de planificar y prepararse para absorber, recuperarse y adaptarse a cualquier evento adverso que pueda suceder, por ejemplo: desastres naturales, por citar sismos, incendios, inundaciones, erupciones volcánicas, huracanes que podrán considerarse de probabilidad baja pero de alto impacto.

Ante estos eventos adversos [14], los sistemas eléctricos deben ser capaces de tolerar estas perturbaciones y continuar con el suministro de electricidad, la resiliencia de un sistema es la capacidad que tiene para recuperarse rápidamente de una gran desconexión o evento a través de cualquier medio para continuar con el suministro de electricidad. En la figura 1 se presenta una clasificación de las causas principales de desconexiones en un sistema.

Hasta la actualidad se han efectuado varias investigaciones partiendo de definir el concepto de resiliencia, entre los cuales se determina que refleja como una infraestructura de un sistema puede moderar las consecuencias de un evento impredecible raro de alto impacto (Black Swan) o de un evento parcialmente predecible raro de alto impacto (Gray Swan) en [15]; en la figura 2 se presenta una curva de comportamiento típico de un sistema ante un incidente.

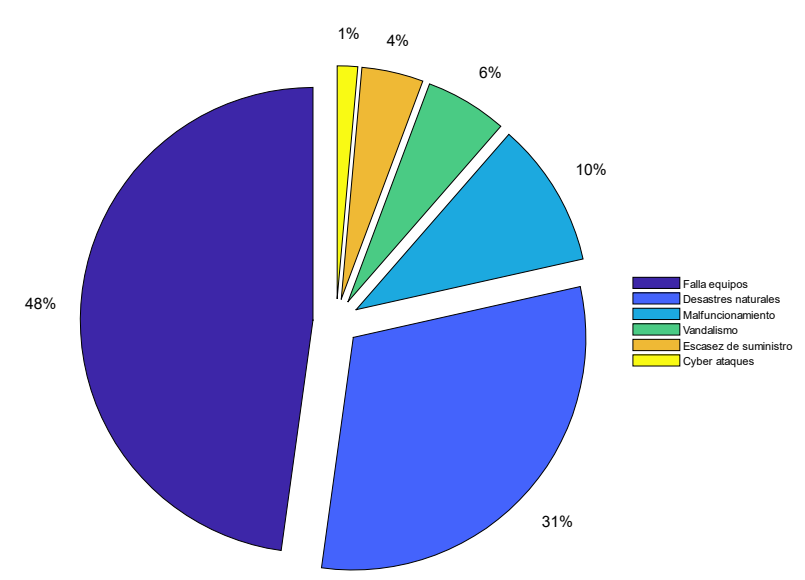

Figura 1. Principales causas de desconexiones.

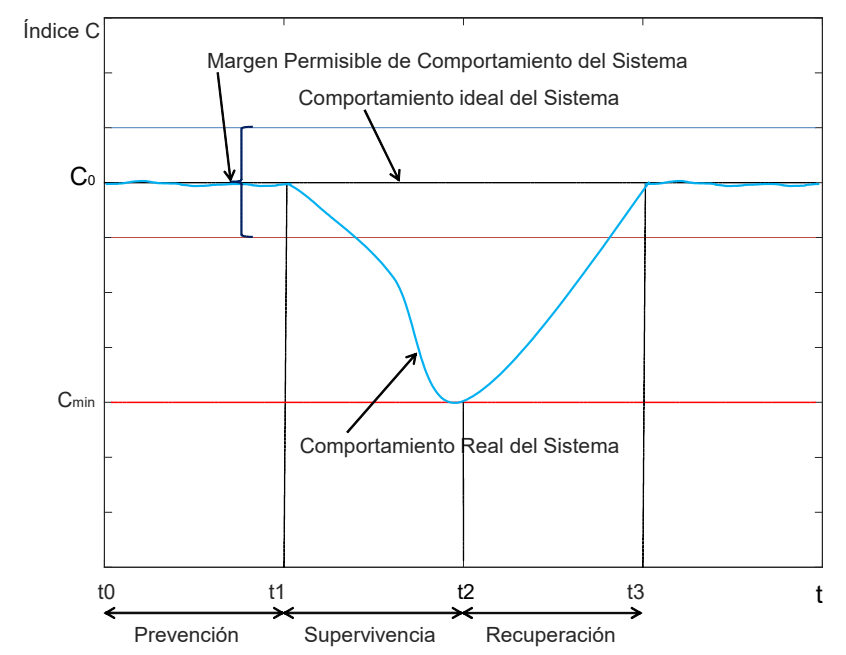

Figura 2. Comportamiento del Sistema de potencia después de un incidente raro de alto impacto.

Por otro lado en [16], se establece que la resiliencia cyber física de un sistema de potencia corresponde a su habilidad para mantener el suministro continuo de electricidad a los clientes bajo un esquema de priorización de carga.

Las métricas de resiliencia que permite disponer de atributos clave son identificados en los cálculos de estabilidad de pequeña señal, cálculos de estabilidad transitoria y el impacto de la latencia de comunicación que permiten determinar un modelo de resiliencia estableciendo una región de confiabilidad [17]; en la figura 3 se presenta un modelo de resiliencia.

En [18], se efectúa una evaluación empírica sobre la resiliencia de la red eléctrica de los EE.UU. soportada en 
la base de datos de eventos de perturbaciones eléctricas, se analiza tres componentes clave asociados con cada apagón y recuperación del sistema de potencia, el tiempo entre interrupciones, pérdida de rendimiento en cada interrupción y el tiempo empleado para la recuperación del sistema, para estos tres componentes se detecta su tendencia a través de la prueba Lewis-Robinson corrigiendo las pruebas estadísticas Military handbook y de Laplace.

A través de una metodología sencilla se evalúa la resiliencia y su interdependencia con subsistemas de la infraestructura de red, el método combina las medidas de resiliencia, modelos de entrada y salida y fragilidades estructurales, según [19].

En [20], recopila 472 contribuciones relacionados a la ingeniería de resiliencia que tiene como objetivo proporcionar herramientas para gestionar proactivamente el riesgo, reconociendo la complejidad inherente al funcionamiento del sistema y la correspondiente necesidad de variabilidad del rendimiento; la definición de métodos para identificar las causas que pueden afectar a la resiliencia de un sistema de energía y sobre todo establecer soluciones que permitan maximizar la resiliencia, sin embargo todavía no se ha definido una receta definitiva.

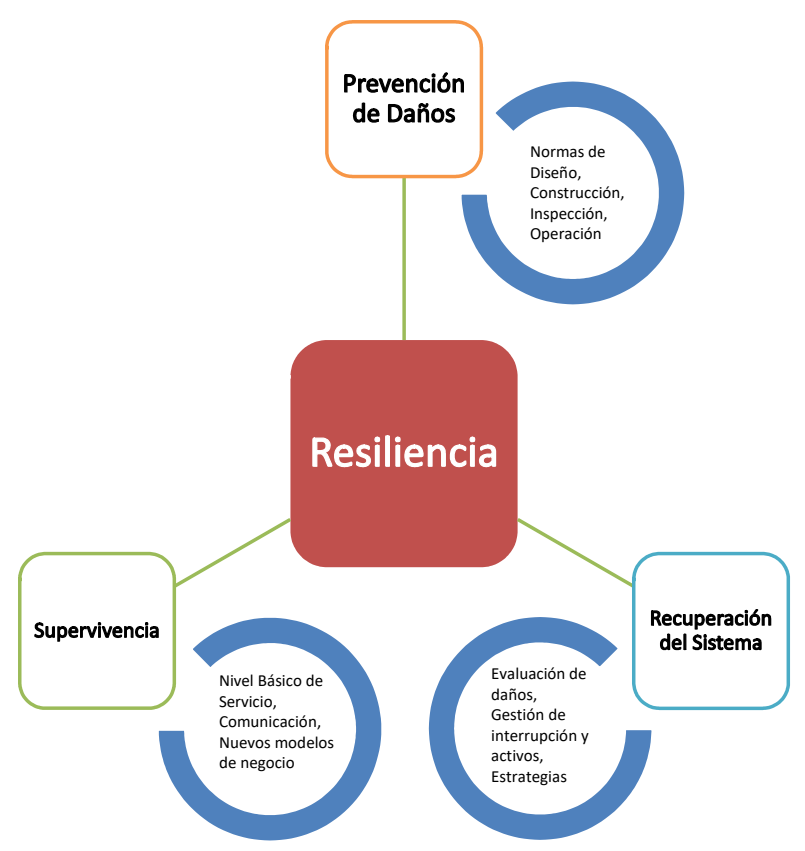

Figura 3. Modelo de Resiliencia.
El sistema eléctrico si bien al inicio de la contingencia puede mantenerse robusto y resistente podrá no ser capaz de soportar la perturbación, para corregir esta situación se plantea el modelado de soluciones que sean robustas a los errores [21], integrando las restricciones de seguridad (contingencia), redes inteligentes, generación distribuida, energías renovables no convencionales, el impacto del clima y la gestión de los riesgos, sin embargo, el concepto de resiliencia en los sistemas de potencia y sus principales amenazas aún no está del todo claro, particularmente en términos de aspectos de modelado y análisis cuantitativos.

En [22], se presenta un modelo de optimización para establecer estrategias secuenciales de operación proactiva, los operadores del sistema tomaran la decisión de ajustar estas estrategias conforme al estado del sistema en tiempo real. Se lo modela con un proceso discreto de decisión en el tiempo de Markov; la estrategia óptima para cada estado del sistema debe asegurar el costo mínimo esperado; en la figura 4 se representa las fases de operación de un sistema, en condiciones normales debería situarse entre normal y emergencia.

De la literatura revisada, se puede destacar estudios que consideran la estimación de estado, modelos de redespacho de generación, la determinación de curvas de fragilidad aplicable a la infraestructura, modelos de regresión de error y de cadena de fallas para determinar líneas sensibles [23], en el Algoritmo 1 se presenta un proceso para determinar el conjunto de líneas sensibles.

Modelos para restauración de sistemas eléctricos que consideran los tiempos de restauración en líneas de transmisión, generalmente se emplean datos históricos de al menos 10 años, con lo cual las estimaciones del tiempo medio de restauración es altamente variable debido a grandes fluctuaciones inherentes a los datos, una buena práctica es el procesamiento automático de la mayor cantidad de datos disponibles para las empresas que operan sistemas de potencia [24].

En la literatura se ha propuesto varios modelos para evaluar el daño en los sistemas de potencia, la duración de la desconexión y restauración después de desastres naturales, sin embargo esta evaluación se la hace después de la ocurrencia del evento extremo. La tarea más importante para el operador del sistema es restaurar el sistema de potencia lo más pronto posible para restablecer las cargas críticas y minimizar las pérdidas 
económicas a los usuarios. Este proceso de restauración puede ser considerado en tres etapas: preparación, restauración del sistema y restablecimiento de la carga, asimismo la generación distribuida puede aportar para mejorar la resiliencia de la red asegurando la disponibilidad de generación en las estrategias de mitigación [25].

Técnicas o metodologías de optimización combinadas se analizan en [26] para la planificación de líneas de transmisión, sin embargo es una tarea compleja debido al problema de la optimización que radica en la exactitud, tiempo de cálculo y confiabilidad, para obtener una solución óptima se encuentra al combinar dos o más técnicas de optimización, algoritmos para gerenciamiento de potencia, sistemas reconfigurables, optimización de topología, modelamiento del clima, optimización del control, gestión de la confiabilidad, sincronización.

El campo de investigación abordado es bastante amplio, y sobre todo acoplar los diversos resultados en una solución única o patrón, no es una tarea fácil, depende de las necesidades de las empresas encargadas de los sistemas eléctricos en función de las acciones a corto y largo plazo que prevean, y en las estrategias que permitan obtener beneficios óptimos para su sustentabilidad.

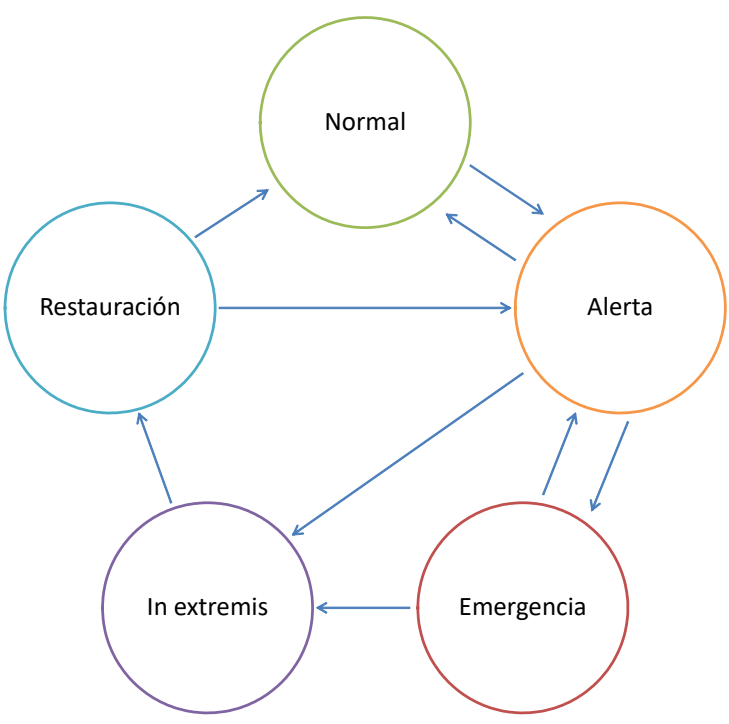

Figura 4. Fases de operación de un sistema.

Para definir el conjunto de líneas sensibles del sistema se debe considerar los siguientes tipos: Tipo 1, la desconexión de las líneas correspondientes, como el segmento inicial de una cadena de falla, causará directamente inestabilidad del sistema; Tipo 2, la desconexión de las líneas correspondientes, como el segmento inicial, seguirá causando inestabilidad del sistema después que se disparen todos los segmentos medios y el nivel de riesgo para la cadena de falla completa es de 4 o 5 .

$\mathrm{La}$ inestabilidad del sistema es causada por varios accidentes (segmentos) en serie, denominado cadena de falla

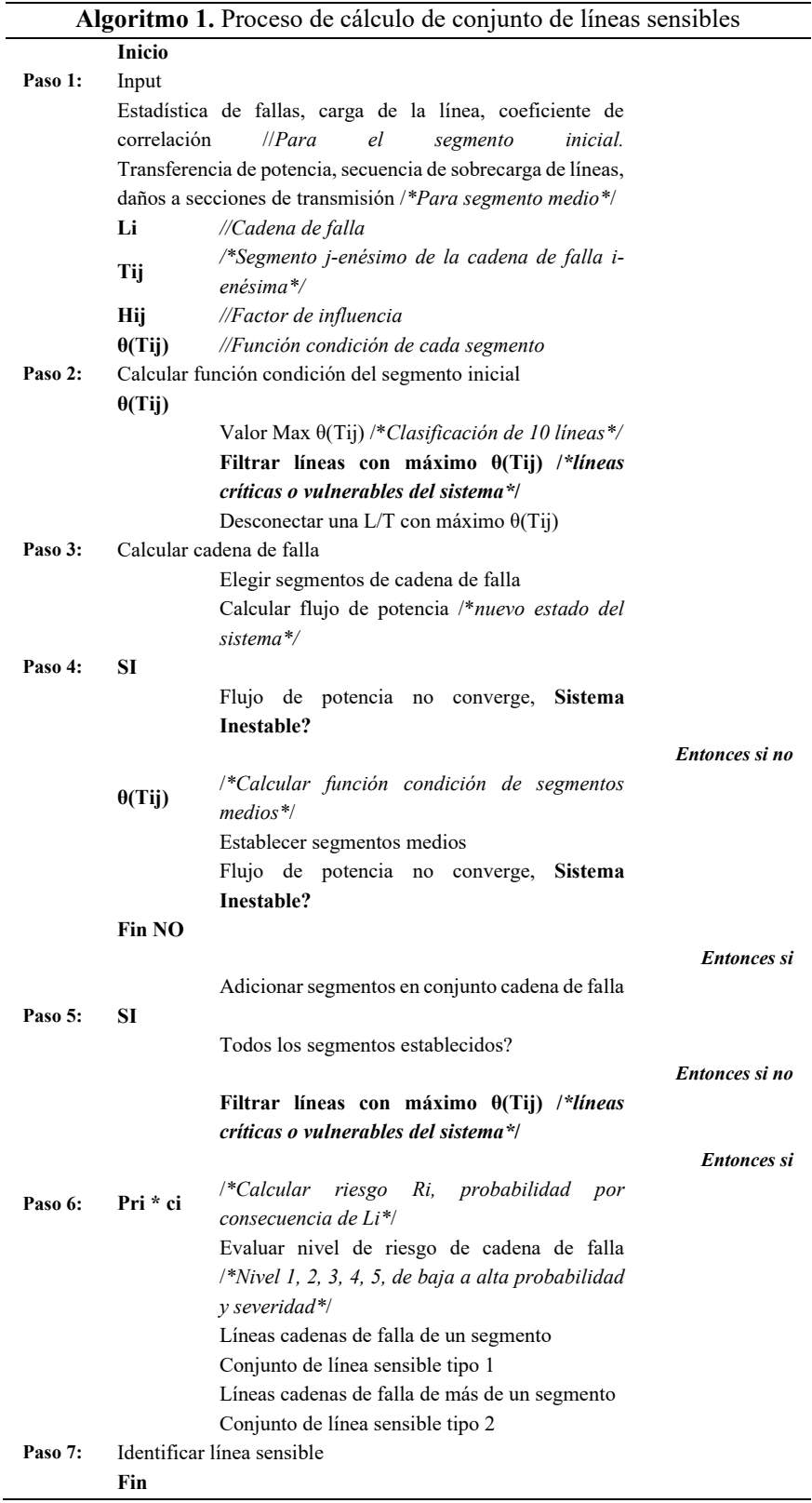

Los artículos enunciados, hasta ahora, consideran el análisis de la resiliencia del sistema eléctrico bajo la 
conceptualización de lo que define a un sistema resiliente.

\subsection{Conmutación de líneas de transmisión}

En esta sección se presenta las contribuciones en el tema de la conmutación de líneas de transmisión, que permitirá comprender la definición del problema.

En [27], la conmutación óptima de líneas de transmisión se considera para reducir costos de generación a través de la desconexión de líneas de transmisión, emplea el modelo de flujo de potencia óptimo DC, clasifica las líneas candidatas a conmutación, posteriormente, a través de una modificación de la heurística en base al DCOPF se usa el flujo de potencia óptimo AC, [28], sin embargo aumenta el tiempo de cómputo siendo poco práctico para sistemas reales, por lo cual aún persisten futuras investigaciones.

Para [29], establece que la conmutación de líneas de transmisión produce un gran ahorro en los costos en especial al limitar el número de líneas a conmutar, el algoritmo implementado como programación de enteros mixtos, presenta mejora en la velocidad de cómputo.

La conmutación en la transmisión ha sido considerada como un método de control para problemas de sobre y bajo voltaje, sobrecargas de líneas de transmisión, reducción de pérdidas y costos, mejora de la seguridad del sistema, por citar algunos; En [30], se aborda el problema de la conmutación óptima de transmisión (OTS), se formula el problema como un programa lineal de enteros mixtos, basado en el flujo óptimo de potencia DC (DCOPF) que se lo usa en el despacho de generadores, emplea variables para representar el estado abierto o cerrado de las líneas de transmisión, sin embargo, se podría usar un flujo óptimo de potencia $\mathrm{AC}$ (ACOPF), condición que requerirá más tiempo de solución debido a la complejidad computacional.

Para el flujo de potencia óptimo básico, la función objetivo, maximiza los costos totales del sistema, sujeta a restricciones físicas del sistema y flujos de potencia.

$$
\mathrm{TC}=\operatorname{Max} \sum_{\mathrm{g}} \mathrm{c}_{\mathrm{ng}} \mathrm{P}_{\mathrm{ng}}
$$

Siendo, $\mathrm{c}_{\mathrm{ng}}$ el costo de generación de electricidad desde el generador $\mathrm{g}$ ubicado en el nodo $\mathrm{n} ; \mathrm{P}_{\mathrm{ng}}$ el flujo de potencia activa hacia o desde el generador $\mathrm{g}$ al nodo $\mathrm{n}$.

Cinco restricciones representan los límites de operación física de la red: el límite del ángulo de voltaje, el límite de capacidad del generador g, el límite del flujo de potencia a través de la línea de transmisión, el balance de potencia en cada nodo, y, las leyes de Kirchhoff.

Para el flujo de potencia óptimo con conmutación óptima de transmisión, la función objetivo maximiza el costo total del sistema con un número $\mathrm{j}$ de líneas de transmisión:

$$
\mathrm{TC}_{\mathrm{j}}=\operatorname{Max} \sum_{\mathrm{k}} \mathrm{c}_{\mathrm{nk}} \mathrm{P}_{\mathrm{nk}}
$$

Siendo, k las líneas de transmisión, la inclusión de restricciones adecuadas fortalece la función objetivo: la quinta restricción se modifica, y se incorpora el límite de líneas abiertas en la red óptima. Como resultados de la simulación en el sistema de prueba de IEEE 118 barras, se evidencia que la conmutación de líneas de transmisión, sin afectar la confiabilidad mejora el despacho económico; sin embargo, podría efectuarse un análisis de contingencias para validar que la seguridad y confiabilidad no se vean afectadas.

En [31], a través de un método secuencial investiga la seguridad del sistema posterior a conmutación óptima de transmisión, considerando el criterio de contingencia $\mathrm{N}$ 1, la formulación del problema OTS y evaluación de la seguridad se basa en el flujo óptimo de potencia AC con un modelo de programación no lineal de enteros mixtos, resultando un incremento del costo total al considerarse las restricciones de seguridad, comparado en algunos casos sin restricciones.

Una solución de conmutación de transmisión, no necesariamente es óptima, pues una modificación de la topología podría afectar las métricas de la seguridad probabilística del sistema, por ende antes de implementar una solución OTS debe realizarse un análisis de seguridad probabilística, [32]; Determinar el conjunto óptimo de líneas a conmutar trae consigo una enorme carga computacional, debido a las formulaciones matemáticas y a las variables binarias que se añaden al modelo, por ende debe tratarse de desarrollar un algoritmo de corte plano que genere desigualdades válidas y variables fijas, [33].

Para [34], la aproximación DC del modelo de flujo de potencia en el problema de conmutación óptima de transmisión puede conducir a soluciones imprecisas y no considerar problemas de estabilidad. Por lo cual se enfoca en el modelo de flujo de potencia AC completo con relajación a través de programación de enteros 
mixtos de segundo orden; sin embargo, la conmutación óptima de transmisión $\mathrm{AC}$ es un problema muy desafiante debido a que presenta al menos dos tipos de no convexidades.

En [35], presenta dos formulaciones matemáticas para conmutación óptima de transmisión, con flujos de potencia óptimos DC y AC, de la comparación el flujo de potencia óptimo AC logra el costo operacional más bajo y con menor tiempo de cómputo que el flujo de potencia óptimo DC.

En [36], a partir del problema de Conmutación Óptima de Transmisión (OTS) que es modelado en base al Flujo Óptimo de Potencia DC (DCOPF), propone un modelo aproximado para el problema OTS. La función objetivo en el modelo OTS (3) tomada de [36], considera minimizar los costos totales de la generación de electricidad sujeto a que: a) la potencia que ingresa y sale de un nodo sea igual; b) los ángulos de voltaje en barras conectadas y el flujo de potencia en líneas conectadas; c) límite térmico de las líneas; d) capacidad de los generadores; e) límites de la diferencia de los ángulos de fase de las barras conectadas; sin embargo, el programa de enteros mixtos presenta dificultad para resolver cuando hay un gran número de líneas de transmisión.

$$
\text { Min, } P_{1}=\sum_{n} C_{n} g_{n}
$$

Donde, $\mathrm{C}_{\mathrm{n}}$ es el costo de operación del generador $\mathrm{n}, \mathrm{y}$, $\mathrm{g}_{\mathrm{n}}$ es la potencia generada por el generador $\mathrm{n}$.

En el modelo aproximado OTS la función objetivo (4) tomada de [36], $\epsilon_{\mathrm{k}}$ es una variable de decisión, y, C' es un número constante.

$$
\text { Min, } P_{3}=\sum_{n} C_{n} g_{n}+C^{\prime} \sum_{k} \frac{\epsilon_{k}}{M_{k}}
$$

Como resultado del análisis efectuado en sistemas de prueba de 14, 39, 57, IEEE 118 y 2383 barras, el modelo aproximado OTS provee similar o el mismo ahorro en el costo, pero, con menor número de líneas de transmisión conmutadas.

Con la finalidad de encontrar la mejor solución al problema de OTS, en [37], se aborda la conmutación de la transmisión con cierta eficiencia económica mientras la seguridad de la red es sujeta a una contingencia N-1. A través de la formulación DCOPF N-1 se asegura que el sistema sobrevivirá a la pérdida de cualquier línea de transmisión o generador, de igual manera, buscando minimizar los costos sujetos a restricciones físicas del sistema y leyes de Kirchhoff de flujos de potencia:

$$
\text { Min: ETC }=\sum_{\mathrm{g}, \mathrm{c}} \rho_{\mathrm{c}} \mathrm{c}_{\mathrm{ngc}} \mathrm{P}_{\mathrm{ngc}}+\sum_{\mathrm{k} \in \mathrm{K}^{\prime}} \mathrm{C}_{\mathrm{k}} \mathrm{z}_{\mathrm{k}}
$$

Siendo: $\rho_{\mathrm{c}}$ la probabilidad de estado de $\mathrm{c}$, cuando $\mathrm{c}=0$ no hay contingencia, para $\mathrm{c}>0$ hay una contingencia $\mathrm{N}-1$; $\mathrm{c}_{\mathrm{ngc}}$ el costo de producción desde el generador $\mathrm{g}$ en el estado c; $\mathrm{P}_{\text {ngc }}$ suministro de potencia desde el generador $\mathrm{g}$ en el nodo $\mathrm{n}$ para el estado $\mathrm{c} ; \mathrm{z}_{\mathrm{k}}$ variable binaria para el elemento de transmisión, 0 abierto y 1 cerrado.

Las restricciones consideran: ángulo del voltaje en el nodo $\mathrm{n}$ para el estado c, balance de potencia en los nodos, límites de líneas de transmisión y generadores para cada estado; debido a la complejidad computacional, se simplifica el modelo con condiciones iniciales de costo cero y sin probabilidad de falla, esto para cuantificar el ahorro con la conmutación de líneas de transmisión ante la contingencia $\mathrm{N}-1$.

Como resultado, se obtiene una reducción de aproximadamente $15 \%$ en el costo sujeto a una contingencia $\mathrm{N}-1$, conmutando líneas de transmisión en el despacho de generación.

$\mathrm{Si}$ bien aún persisten desafíos que no han sido estudiados a profundidad, por citar: la complejidad computacional al manejar un modelo completo, la incorporación de sistemas reales a gran escala, la evaluación de la estabilidad dinámica del sistema y la aplicación de ACOPF, han permitido que [38] considere una corrección $\mathrm{AC}$ a la conmutación de transmisión en base al análisis de contingencias en tiempo real, lo cual usa datos del estimador de estado y del análisis de contingencias en flujos de potencia AC.

El análisis de contingencias en tiempo real RTCA, no considera ninguna optimización, no impone ninguna restricción, su finalidad es identificar contingencias críticas que puedan afectar la confiabilidad del sistema.

Con respecto a la estabilidad del sistema eléctrico, las contingencias, la conmutación de líneas de transmisión asocian cambios importantes en la estabilidad del sistema, lo que podría volverlo vulnerable haciéndolo inestable; siendo esta condición la causa de varios apagones.

La estabilidad del sistema evalúa únicamente si la conmutación de transmisión obtenida es estable. La simulación en el dominio del tiempo considera las contingencias en líneas de transmisión y en generadores. 
Se identifica que la conmutación de transmisión, reduciría los costos operativos, mejoraría la confiabilidad del sistema y mejoraría la incorporación de recursos renovables. En el Algoritmo 2 se presenta el procedimiento RTCA.

\begin{tabular}{|c|c|c|c|}
\hline \multicolumn{4}{|c|}{ Algoritmo 2. Procedimiento para RTCA con CTS } \\
\hline \multirow{3}{*}{ Paso 1: } & Inicio & & \\
\hline & Input & & \\
\hline & ACOPF & $\begin{array}{l}\text { /*Información del estado del sistema } y \\
\text { topología*/ }\end{array}$ & \\
\hline Paso 2: & \multicolumn{3}{|c|}{ Ejecutar CA //Análisis de contingencia } \\
\hline Paso 3: & \multicolumn{3}{|c|}{ Resultados CA //Mostrar resultados } \\
\hline Paso 4: & \multicolumn{3}{|c|}{ Identificar contingencias críticas } \\
\hline Paso 5: & \multicolumn{3}{|c|}{ Mostar $\mathrm{c}=1 / /$ Contingencia $c(N-1)$} \\
\hline Paso 6: & \multicolumn{3}{|c|}{ Generar lista de elementos de conmutación } \\
\hline Paso 7: & \multicolumn{3}{|c|}{$\begin{array}{l}\text { Comprobar reducción de violaciones } /{ }^{*} \text { Conmutación de } \\
\text { transmisión*/ }\end{array}$} \\
\hline Paso 8: & \multicolumn{3}{|c|}{ Seleccionar 5 soluciones de conmutación } \\
\hline \multirow[t]{11}{*}{ Paso 9: } & SI & & \\
\hline & & Contingencias críticas verificadas? & \\
\hline & & & Entonces si no \\
\hline & & For $\mathrm{c}=\mathrm{c}+1 / /$ Contingencia $c(N-1-1)$ & \\
\hline & & Generar lista de elementos de conmutación & \\
\hline & & Comprobar reducción de violaciones & \\
\hline & & /*Conmutación de transmisión*/ & \\
\hline & & Seleccionar 5 soluciones de conmutación & \\
\hline & & & Entonces si \\
\hline & \multicolumn{3}{|l|}{ Fin SI } \\
\hline & \multicolumn{3}{|l|}{ Fin } \\
\hline
\end{tabular}

Para [39], la conmutación óptima de transmisión se emplea como una herramienta para manejar la congestión en la red eléctrica, cambiando la topología. La conmutación de transmisión se formula como un problema de optimización; para aliviar la congestión se considera la seguridad de voltaje, se desarrolla a través de flujos óptimos de potencia con restricciones AC y variables binarias.

Una metodología para manejar la congestión usando conmutación óptima de transmisión considera la minimización del costo total de operación y la maximización de la confiabilidad, incluye la posibilidad de cambio de topología existente, como resultados de obtiene un ahorro substancial sin afectar la confiabilidad del sistema se describe en [40].

\subsection{Conmutación de líneas en planificación de transmisión}

En [41], el problema tradicional de la planificación de la expansión de transmisión (TEP) es minimizar el costo de inversión de la línea de transmisión, sin embargo un objetivo ideal sería el bienestar social. Considera la siguiente función objetivo:

$$
\begin{aligned}
& \operatorname{Min} C=\sum_{\mathrm{t}=1}^{\mathrm{TPL}} \sum_{\mathrm{k} \in \Omega_{\mathrm{k}}} \frac{\mathrm{c}_{\mathrm{k}}\left(\mathrm{z}_{\mathrm{kt}}-\mathrm{z}_{\mathrm{kt}-1}\right)}{(1+\mathrm{d})^{\mathrm{t}-1}} \\
& +\sum_{\mathrm{t}=1}^{\mathrm{TOP}} \sum_{\mathrm{g} \in \Omega_{\mathrm{g}}} \frac{\mathrm{CG}_{\mathrm{gt}}}{(1+\mathrm{d})^{\mathrm{t}-1}}
\end{aligned}
$$

Donde, el primer término representa el costo total de planificación, el segundo término representa el costo total de operación del generador; $c_{k}$ es el costo de construir la línea $\mathrm{k}, \mathrm{z}_{\mathrm{kt}}$ es una variable de decisión binaria para determinar si se construye o no la línea; $\mathrm{c}_{\mathrm{k}}\left(\mathrm{z}_{\mathrm{kt}}-\mathrm{z}_{\mathrm{kt}}\right.$ 1) garantiza que $c_{k}$ es una inversión única; la variable inicial de decisión $\mathrm{z}_{\mathrm{k} 0}$ es cero. TPL y TOP son los horizontes totales de planificación y operación anual, respectivamente; $\mathrm{CG}_{\mathrm{gt}}$ corresponde el costo de energía del generador; $\mathrm{d}$ es un factor de descuento.

Las restricciones del problema entre otras consideran el balance de potencia en cada nodo, si una línea existe o es seleccionada para construirse, el flujo máximo a través de la línea, la diferencia angular entre dos nodos, el límite de generación programada.

Para [42], la planificación de la expansión usando conmutación de transmisión la define como un problema principal o maestro de plan óptimo de inversión, que se complementa con dos problemas para verificación de la seguridad y la operación óptima. Un conjunto típico de restricciones de planificación consideran: fondos de inversión de capital, recursos proyectados y capacidad de la línea, número máximo de unidades de generación y líneas de transmisión a añadirse, tiempo de construcción de la inversión candidata; por otro lado las restricciones de operación consideran: balance de potencia, límites de flujo por líneas de transmisión, límites de generación; es decir, minimiza el costo total del sistema mientras satisface las restricciones de seguridad y confiabilidad del sistema.

En [43], el objetivo de la formulación TEP es encontrar una estructura de transmisión óptima para satisfacer la demanda de carga máxima con mínima inversión y pérdidas de costo de carga, mientras se satisfacen las limitaciones operativas.

En [44], a partir del modelo matemático para planificación básica que usa un modelo DC sin considerar la incertidumbre de la demanda, se plantean dos modelos que consideran la incertidumbre en la demanda total e individual en cada barra de carga, de esta 
forma la demanda se considera como una variable del problema ya no es un dato.

$$
\begin{gathered}
\text { Min } v=\sum_{(\mathrm{i}, \mathrm{j}) \in \Omega} \mathrm{c}_{\mathrm{ij}} \mathrm{n}_{\mathrm{ij}} \\
\operatorname{Min} \mathrm{v}=\sum_{(\mathrm{i}, \mathrm{j}) \in \Omega} \mathrm{c}_{\mathrm{ij}} \mathrm{n}_{\mathrm{ij}}+\alpha \sum \mathrm{r}_{\mathrm{i}}-\beta \mathrm{z} \\
\operatorname{Minv}=\sum_{(\mathrm{i}, \mathrm{j}) \in \Omega} \mathrm{c}_{\mathrm{ij}} \mathrm{n}_{\mathrm{ij}}+\alpha \sum \mathrm{r}_{\mathrm{i}}-\delta \sum \mathrm{d}_{\mathrm{i}}
\end{gathered}
$$

Donde, $\mathrm{v}$ es la inversión; $\mathrm{c}_{\mathrm{ij}}, \mathrm{n}_{\mathrm{ij}}$ representan el costo de un circuito y el número de circuitos que pueden ser añadidos al derecho de paso $\mathrm{i}-\mathrm{j}$; $\mathrm{r}$ es el vector de desconexión de carga; $\alpha$ es un parámetro de penalización en la función objetivo por desconexión de carga del sistema; $\beta$ (USD/MW) es el parámetro que maximiza la demanda suministrada en el sistema; $d_{i}$ es la demanda en cada barra de carga i; $\delta$ (USD/MW) es el parámetro que maximiza la demanda suministrada en cada barra en el sistema; $\Omega$ es el conjunto de todos los derechos de paso.

En si con el problema de TEP, podría obtenerse una planificación más barata que satisfaga toda la demanda que podría incrementarse en el futuro, evitando una expansión sobre dimensionada del sistema.

\section{Resultados}

La aplicación de los modelos determinados en la revisión literaria, determinando las restricciones que permitan alcanzar la solución óptima sin penalizar la respuesta computacional, permitirán demostrar que una red puede satisfacer el estándar N-1 mientras se reducen los costos incorporando la conmutación de líneas de transmisión, favoreciendo así el despacho económico de generación, en [37] a través de los sistemas de pruebas, se obtuvo hasta un ahorro de $15 \%$ en el costo de generación con un modelo N-1 DCOPF debido a la conmutación de la transmisión.

Para [39], la administración de la congestión de la transmisión usando conmutación de líneas de transmisión considerando criterios $\mathrm{N}-1$ y seguridad de voltaje, permitió determinar las líneas más idóneas a ser desconectadas para aliviar la congestión, sin embargo, la seguridad del sistema juega un rol importantísimo que puede comprometer la remoción de la congestión del sistema.

Para [41-44], si bien en esencia los modelos determinan la expansión del sistema a través de la construcción o no de una línea de transmisión o de un generador, en los diferentes resultados para un sistema en específico, las líneas o generadores candidatos no siempre pueden coincidir debido a la complejidad de los modelos a través de las restricciones.

Los modelos previstos por los diversos autores, son resueltos mediante programación lineal entera mixta, las funciones objetivo se enfocan en la minimización de los costos de operación, se plantea la metodología de los flujos óptimos de potencia en DC y AC, siendo el primero menos complejo y computacionalmente más rápido, su simplicidad permite tener un margen aceptable de precisión, posteriormente al incorporar los criterios de seguridad y estabilidad del sistema analizando contingencias $\mathrm{N}-1$, se prevén estrategias que puedan ser implementadas por los operadores de los sistemas eléctricos.

Asimismo, se espera que los potenciales beneficios de la conmutación óptima de transmisión y sus efectos en la estabilidad, confiabilidad y seguridad del servicio del sistema no decrezcan, estableciéndose como un sistema resiliente ante contingencias $\mathrm{N}-1$.

El problema TEP podría considerarse en el horizonte de la operación del sistema de transmisión, dándole un enfoque adecuado en el aspecto de la resiliencia a través del restablecimiento del sistema después de una contingencia, evaluando líneas candidatas existentes para ser conectadas o desconectadas.

\section{Conclusiones}

Las investigaciones actuales han contribuido para establecer una definición de resiliencia en un sistema de potencia, a pesar que no se ha determinado una metodología o identificado métricas óptimas que permitan cuantificar la resiliencia en un sistema eléctrico, se requiere disponer de una visión clara del estado del sistema antes, durante y después de ocurrido un evento extremo severo.

Cuantificar la resiliencia depende de la interrelación con otros sistemas y factores sociales, económicos y de infraestructura. Se han propuesto varios modelos que abordan la problemática, por citar cadenas de Markov, simulación de Monte-Carlo, de regresión, de cadena de 
falla, de optimización, etc. Todos en busca de las mejores soluciones que permitan obtener beneficios comprobados, sin embargo, se han obtenido buenos resultados sin alcanzar lo óptimo.

Se han identificado estrategias de mejora en lo que respecta a la capacidad operacional y de recuperación de la infraestructura, donde se considera un rol importante la concientización de los operadores del sistema, contrastado con la intención de que casi no exista intervención humana, para que el sistema se adapte y recupere ante cualquier evento.

La óptima conmutación de líneas de transmisión que permitan maximizar la resiliencia del sistema eléctrico, presenta aspectos atractivos en función de minimizar el costo total de despacho; en la planificación y operación de los sistemas eléctricos, el modelamiento permitirá determinar las líneas y sus tramos sensibles, determinar las líneas candidatas para conmutación, cuantificar el flujo de potencia, optimizar el despacho de generación y mantener la mayor cantidad de carga.

Las investigaciones citadas presentan buenos resultados, por lo tanto su metodología es aplicable en la restauración del sistema eléctrico a través de la conmutación de líneas de transmisión, validando las restricciones físicas, de tal forma que los modelos permitan obtener resultados confiables en las simulaciones.

\section{REFERENCIAS}

[1] R. S. Liévanos and C. Horne, "Unequal resilience: The duration of electricity outages," Energy Policy, vol. 108, no. August 2016, pp. 201-211, 2017.

[2] M. Panteli and P. Mancarella, "Influence of extreme weather and climate change on the resilience of power systems: Impacts and possible mitigation strategies," Electr. Power Syst. Res., vol. 127, pp. 259-270, 2015.

[3] M. Panteli, P. Mancarella, D. N. Trakas, E. Kyriakides, and N. D. Hatziargyriou, "Metrics and Quantification of Operational and Infrastructure Resilience in Power Systems," IEEE Trans. Power Syst., vol. 32, no. 6, pp. 4732-4742, 2017.

[4] M. Panteli, C. Pickering, S. Wilkinson, R. Dawson, and P. Mancarella, "Power System Resilience to Extreme Weather: Fragility Modeling, Probabilistic Impact Assessment, and Adaptation Measures," IEEE Trans. Power Syst., vol. 32, no. 5, pp. 3747-3757, 2017.

[5] M. Panteli, D. N. Trakas, P. Mancarella, and N. D. Hatziargyriou, "Power Systems Resilience Assessment: Hardening and Smart Operational Enhancement Strategies," Proc. IEEE, vol. 105, no. 7, pp. 1202-1213,
2017.

[6] G. Huang, J. Wang, C. Chen, J. Qi, and C. Guo, "Integration of Preventive and Emergency Responses for Power Grid Resilience Enhancement," IEEE Trans. Power Syst., vol. 32, no. 6, pp. 4451-4463, 2017.

[7] P. Dehghanian, S. Aslan, and P. Dehghanian, "Maintaining Electric System Safety Through An Enhanced Network Resilience," IEEE Trans. Ind. Appl., vol. 54, no. 5, pp. 4927-4937, 2018.

[8] L. Molyneaux, L. Wagner, C. Froome, and J. Foster, "Resilience and electricity systems: A comparative analysis," Energy Policy, vol. 47, pp. 188-201, 2012.

[9] J. Wang and H. Gharavi, "Power Grid Resilience [Scanning the Issue]," Proc. IEEE, vol. 105, no. 7, pp. 1199-1201, 2017.

[10] S. Zhao and C. Singh, "A hybrid method for reliability evaluation of line switching operations," Electr. Power Syst. Res., vol. 163, no. June, pp. 365-374, 2018.

[11] M. T. Craig et al., "A review of the potential impacts of climate change on bulk power system planning and operations in the United States," Renew. Sustain. Energy Rev., vol. 98, no. August, pp. 255-267, 2018.

[12] C. Varianou Mikellidou, L. M. Shakou, G. Boustras, and C. Dimopoulos, "Energy critical infrastructures at risk from climate change: A state of the art review," Saf. Sci., no. December, pp. 0-1, 2017.

[13] A. Sharifi and Y. Yamagata, "Principles and criteria for assessing urban energy resilience: A literature review," Renew. Sustain. Energy Rev., vol. 60, pp. 1654-1677, 2016.

[14] Z. Bie, Y. Lin, G. Li, and F. Li, "Battling the Extreme: A Study on the Power System Resilience," Proc. IEEE, vol. 105, no. 7, pp. 1253-1266, 2017.

[15] A. Gholami, T. Shekari, M. H. Amirioun, F. Aminifar, M. H. Amini, and A. Sargolzaei, "Toward a consensus on the definition and taxonomy of power system resilience," IEEE Access, vol. 6, pp. 32035-32053, 2018.

[16] R. Arghandeh, A. Von Meier, L. Mehrmanesh, and L. Mili, "On the definition of cyber-physical resilience in power systems," Renew. Sustain. Energy Rev., vol. 58, pp. 1060 1069, 2016.

[17] K. Eshghi, B. K. Johnson, and C. G. Rieger, "Metrics required for power system resilient operations and protection," Proc. - 2016 Resil. Week, RWS 2016, pp. 200203, 2016.

[18] L. Shen, B. Cassottana, and L. C. Tang, "Statistical trend tests for resilience of power systems," Reliab. Eng. Syst. Saf., vol. 177, no. May, pp. 138-147, 2018.

[19] D. A. Reed, K. C. Kapur, and R. D. Christie, "Methodology for assessing the resilience of networked infrastructure," IEEE Syst. J., vol. 3, no. 2, pp. 174-180, 2009.

[20] R. Patriarca, J. Bergström, G. Di Gravio, and F. Costantino, "Resilience engineering: Current status of the research and future challenges," Saf. Sci., vol. 102, no. August 2017, pp. 79-100, 2018.

[21] M. Panteli and P. Mancarella, "Modeling and evaluating the 
resilience of critical electrical power infrastructure to extreme weather events," IEEE Syst. J., vol. 11, no. 3, pp. 1733-1742, 2017.

[22] C. Wang, Y. Hou, F. Qiu, S. Lei, and K. Liu, "Resilience Enhancement with Sequentially Proactive Operation Strategies," IEEE Trans. Power Syst., vol. 32, no. 4, pp. 2847-2857, 2017.

[23] J. Yang and K. Jiang, "The sensitive line identification in resilient power system based on fault chain model," Int. J. Electr. Power Energy Syst., vol. 92, pp. 212-220, 2017.

[24] S. Kancherla and I. Dobson, "Heavy-tailed transmission line restoration times observed in utility data," IEEE Trans. Power Syst., vol. 33, no. 1, pp. 1145-1147, 2018.

[25] Y. Wang, C. Chen, J. Wang, and R. Baldick, "Research on Resilience of Power Systems under Natural Disasters - A Review," IEEE Trans. Power Syst., vol. 31, no. 2, pp. 16041613, 2016.

[26] T. S. Kishore and S. K. Singal, "Optimal economic planning of power transmission lines: A review," Renew. Sustain. Energy Rev., vol. 39, pp. 949-974, 2014.

[27] J. D. Fuller, R. Ramasra, and A. Cha, "Fast heuristics for transmission-line switching," IEEE Trans. Power Syst., vol. 27, no. 3, pp. 1377-1386, 2012.

[28] M. Soroush and J. D. Fuller, "Accuracies of optimal transmission switching heuristics based on DCOPF and ACOPF," IEEE Trans. Power Syst., vol. 29, no. 2, pp. 924 932, 2014.

[29] C. Barrows and S. Blumsack, "Transmission Switching in the RTS-96 Test System," IEEE Trans. Power Syst., vol. 27, no. 2, pp. 1134-1135, 2011.

[30] E. B. Fisher, R. P. O’Neill, and M. C. Ferris, "Optimal Transmission Switching," IEEE Trans. Power Syst., vol. 23, no. 3, pp. 1346-1355, 2008.

[31] M. Tarafdar Hagh, M. Zamani Gargari, and M. J. Vahid Pakdel, "Sequential analysis of optimal transmission switching with contingency assessment," IET Gener. Transm. Distrib., vol. 12, no. 6, pp. 1390-1396, 2017.

[32] P. Henneaux and D. S. Kirschen, "Probabilistic security analysis of optimal transmission switching," IEEE Trans. Power Syst., vol. 31, no. 1, pp. 508-517, 2016.

[33] J. Ostrowski, J. Wang, and C. Liu, "Transmission switching with connectivity-ensuring constraints," IEEE Trans. Power Syst., vol. 29, no. 6, pp. 2621-2627, 2014.
[34] B. Kocuk, S. S. Dey, and X. A. Sun, "New Formulation and Strong MISOCP Relaxations for AC Optimal Transmission Switching Problem," vol. 32, no. 6, pp. 1-25, 2016.

[35] T. Lan, Z. Zhou, and G. M. Huang, "Modeling and Numerical Analysis of Stochastic Optimal Transmission Switching with DCOPF and ACOPF," IFACPapersOnLine, vol. 51, no. 28, pp. 126-131, 2018.

[36] M. Jabarnejad, "Approximate optimal transmission switching," Electr. Power Syst. Res., vol. 161, pp. 1-7, 2018.

[37] K. W. Hedman, R. P. O’Neill, E. B. Fisher, and S. S. Oren, "Optimal Transmission Switching With Contingency Analysis," IEEE Trans. Power Syst., vol. 24, no. 3, pp. 1577-1586, 2009.

[38] X. Li, P. Balasubramanian, M. Sahraei-Ardakani, M. AbdiKhorsand, K. W. Hedman, and R. Podmore, "Real-Time Contingency Analysis with Corrective Transmission Switching," IEEE Trans. Power Syst., vol. 32, no. 4, pp. 2604-2617, 2017.

[39] M. Khanabadi, H. Ghasemi, and M. Doostizadeh, "Optimal transmission switching considering voltage security and N1 contingency analysis," IEEE Trans. Power Syst., vol. 28, no. 1, pp. 542-550, 2013.

[40] S. R. Salkuti, "Congestion management using optimal transmission switching," IEEE Syst. J., vol. 12, no. 4, pp. 3555-3564, 2018

[41] H. Zhang, V. Vittal, G. T. Heydt, and J. Quintero, “A mixedinteger linear programming approach for multi-stage security-constrained transmission expansion planning," IEEE Trans. Power Syst., vol. 27, no. 2, pp. 1125-1133, 2012.

[42] A. Khodaei, M. Shahidehpour, and S. Kamalinia, "Transmission switching in expansion planning," IEEE Trans. Power Syst., vol. 25, no. 3, pp. 1722-1733, 2010.

[43] G. A. Orfanos, P. S. Georgilakis, and N. D. Hatziargyriou, "Transmission expansion planning of systems with increasing wind power integration," IEEE Trans. Power Syst., vol. 28, no. 2, pp. 1355-1362, 2013.

[44] I. de J. Silva, M. J. Rider, R. Romero, and C. A. F. Murari, "Transmission network expansion planning considering uncertainty in demand," IEEE Trans. Power Syst., vol. 21, no. 4, pp. 1565-1573, 2006. 